\title{
Development of a Novel Preparation Method for Highly Porous Heat-Resistant Rigid Polymer Network Films and Evaluation of their Properties
}

\author{
Tetsuya Uchida*, Ryo Ikeda, Tomoaki Suzuki and Ryotaro Nakayama \\ Graduate School of Natural Science and Technology, \\ Okayama University, 3-1-1 Tsushima-naka, Kita-ku Okayama 700-8530, Japan \\ tuchida@cc.okayama-u.ac.jp
}

\begin{abstract}
A rigid polymer network of poly(p-phenylene benzobisoxazole) (PBO) has excellent mechanical properties and thermal stability. Many nano voids exist within the network. However, a PBO network cannot be formed into films or fibers. Generally, PBO networks are prepared by one-step polycondensation in polyphosphoric acid. In this study, we have prepared a PBO network by a two-step method involving polymerization of a PBO network precursor (pre-PBO network) in $N$-methyl-2-pyrrolidone followed by thermal cyclization. Heat-resistant porous PBO network films have been successfully obtained by this two-step method. The properties of the PBO network films have been evaluated.
\end{abstract}

Keywords: rigid polymer, poly(p-phenylene benzobisoxazole), network polymer, polycondensation, film

\section{Introduction}

$\operatorname{Poly}(p$-phenylene benzobisoxazole) $(\mathrm{PBO})$ is a rigid polymer. Its molecular chain is resistant to folding due to the linear linkage of benzene and oxazole rings, as shown in Fig. 1. PBO fibers are reported to possess high mechanical strength, high modulus, and high heat resistance [1]. In the synthesis of PBO, branches and cross-linking points are introduced in the molecular chain by the addition of trifunctional monomers so as to generate a network in which the branch points are linked together by linear rigid PBO components, as shown in Fig. 2 [2]. Nanometer-scale voids exist in the structure, and high-performance functional materials are expected to be produced. However, PBO network prepared by previous methods have shown extremely poor processability, such that molding and the fabrication of fibers and thin films proved difficult. To address this issue, a novel preparation method for PBO network film has been developed in the present study, which involves a precursor material. On the same reason, the processability of linear $\mathrm{PBO}$ has been improved [3, 4]. A flexible precursor of the PBO network was synthesized and the material was molded at this stage. A second polymerization step was performed to prepare a PBO network by a cyclization reaction. Salient properties of the prepared PBO network films have been evaluated.

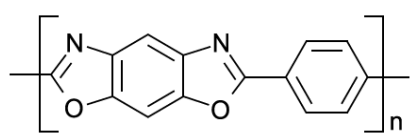

Fig. 1. Poly ( $p$-phenylene benzobisoxazole) (PBO).

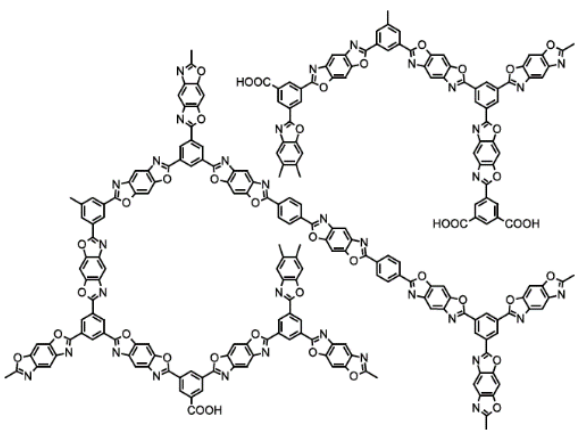

Fig. 2. Model structure of PBO network.

\section{Experimental}

2.1. Preparation of PBO network 
2.1.1. Synthesis of 4,6-di(trimethylsilylamino)-1, 3-di(trimethylsiloxy)benzene (TMS-DAR)

Under a nitrogen atmosphere, 4,6-diaminoresorcinol dihydrochloride (DAR; $5.000 \mathrm{~g}$ ) was placed in a $200 \mathrm{~mL}$, two-necked flask and $N$-methyl-2-pyrrolidone (NMP; $53 \mathrm{~mL}$ ) was added as a solvent. Under nitrogen flow, pyridine $(4.850 \mathrm{~g}, 187.7 \mathrm{mmol})$ and chlorotrimethylsilane $(15.297 \mathrm{~g}, 140.8 \mathrm{mmol})$ were added. TMS-DAR was synthesized by stirring the solution at room temperature for $1 \mathrm{~h}$ and at $60^{\circ} \mathrm{C}$ in an oil bath for $4 \mathrm{~h}$.

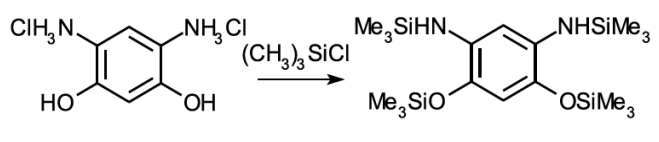

DAR TMS-DAR

Fig. 3. Synthesis of silylated 4,6-diaminoresorcinol (TMS-DAR).

\subsubsection{Preparation of $P B O$ network film}

A mixture of TMS-DAR, terephthaloyl chloride (TPC), and 1,3,5-benzenetricarbonyl trichloride (TMC) in NMP was poured into a molding vessel. As a result, a flexible PBO network precursor (pre-PBO network) film linked by amide bonds was obtained. The obtained pre-PBO network film was then heated to induce cyclization to form an oxazole ring. In this way, PBO network film was obtained, as shown in Fig. 4.

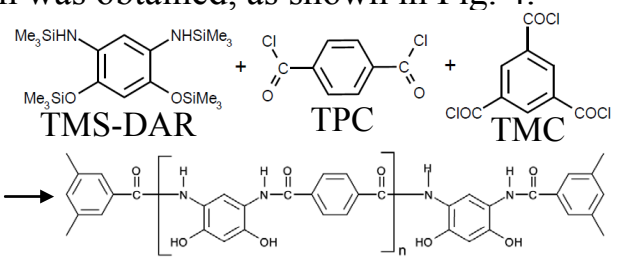

PBO network precursor (pre-PBO network)

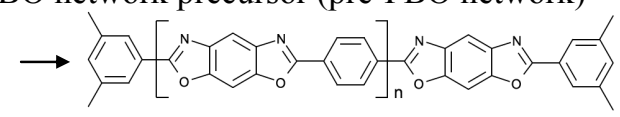

PBO network

Fig. 4. New synthesis route for PBO network.

2.2. Evaluation of physical properties

2.2.1 Infrared (IR) absorption spectrum of the PBO network film

The IR spectra of the pre-PBO and $\mathrm{PBO}$ networks were measured from samples in $\mathrm{KBr}$ on an IRAffinity-1 spectrometer (Shimadzu Corporation).

2.2.2. Apparent density measurement and porosity calculation of the PBO network film
The apparent density was evaluated from the volume and weight of the obtained PBO network. In addition, the porosity was calculated based on the density of PBO fiber [5].

2.2.3. Surface and fracture surface observation of the PBO network film

The surface and fracture surface of the PBO network film were observed by means of a JSM-6320F (JEOL Ltd.) scanning electron microscope.

\subsubsection{Micropore measurement of the PBO} network film

The carbon dioxide adsorption isotherm was measured at $298 \mathrm{~K}$ on a BELSORP-max (BEL Japan, Inc.). The micropore capacity was calculated from the results obtained.

\subsubsection{Thermogravimetric analysis (TGA) of the PBO network film}

TGA of the PBO network film was performed at a heating rate of $10{ }^{\circ} \mathrm{C} / \mathrm{min}$ to $800^{\circ} \mathrm{C}$ under a nitrogen flow rate of $100 \mathrm{~mL} / \mathrm{min}$ on a Thermo Plus TG8 120/SD thermogravimetric analyzer (Rigaku Corporation).

\subsubsection{Heat diffusivity measurement of the PBO network film}

Heat diffusivity was measured using Thermowave Analyzer (Bethel Co., Ltd.).

\subsubsection{Thermal coefficient of linear expansion measurement of the PBO network film}

The thermal coefficient of linear expansion of the PBO network film was measured on a TMA/SS120 apparatus (Seiko Instruments Inc.). The measurement was performed with a load of 5 gf $(49 \mathrm{mN})$, a sample of width ca. $4 \mathrm{~mm}$ and length ca. $10 \mathrm{~mm}$ between chucks, and a nitrogen flow rate of $50 \mathrm{~mL} / \mathrm{min}$.

\subsubsection{Mechanical property test of the PBO network film}

The prepared PBO network was subjected to a bending test at a rate of $4 \mathrm{~mm} / \mathrm{min}$ on an SV-201NA tensile/compression testing machine (Imada Seisakusyo Co., Ltd.). The sample used had length $20 \mathrm{~mm}$ and width $5 \mathrm{~mm}$.

\subsubsection{Dynamic viscoelasticity measurement of the PBO network}

The dynamic viscoelasticity of the PBO network was measured from room temperature to $400{ }^{\circ} \mathrm{C}$ 
in air using a RHEOVIBRON DDV-01FP-AO (ORENTEC Co., LTD.).

\subsubsection{Heat treatment of the PBO network film}

The prepared PBO network film was heated to $450{ }^{\circ} \mathrm{C}$ over a period of $1.5 \mathrm{~h}$ and kept at this temperature for $8 \mathrm{~h}$ using an FT-101VAC electric furnace (Full-Tech Corporation). TGA of the heat-treated sample was then performed by heating from room temperature to $800{ }^{\circ} \mathrm{C}$ at a rate of $10^{\circ} \mathrm{C} / \mathrm{min}$ under nitrogen flow at $100 \mathrm{~mL} / \mathrm{min}$ on a Thermo Plus TG8 120/SD thermogravimetric analyzer.

\section{Results and Discussion}

\subsection{Preparation of PBO network film}

A red solution of TMS-DAR was obtained by silylation of DAR in NMP. By introducing TPC as a bifunctional monomer and TMC as a trifunctional monomer into this solution, a gelation could proceed to afford a red pre-PBO network. Through optimization of the solvent, temperature, and the ratio of bifunctional to trifunctional monomer in the synthesis of the pre-PBO network, the processability of the product could be improved. The optimized ratio of TPC to TMC was identified as 7:3, and the optimal monomer concentration was $0.14 \mathrm{~mol} / \mathrm{L}$. Unless mentioned otherwise, all of the PBO networks described hereinafter were synthesized with this composition.

Following the gelation, the pre-PBO was purified by washing with NMP and distilled water. The pre-PBO was molded at $170{ }^{\circ} \mathrm{C}$ for $30 \mathrm{~min}$ using a vacuum heating press to produce a thin-film pre-PBO network. The IR spectrum of the film featured a peak due to an amide bond, which was characteristic of the pre-PBO network.

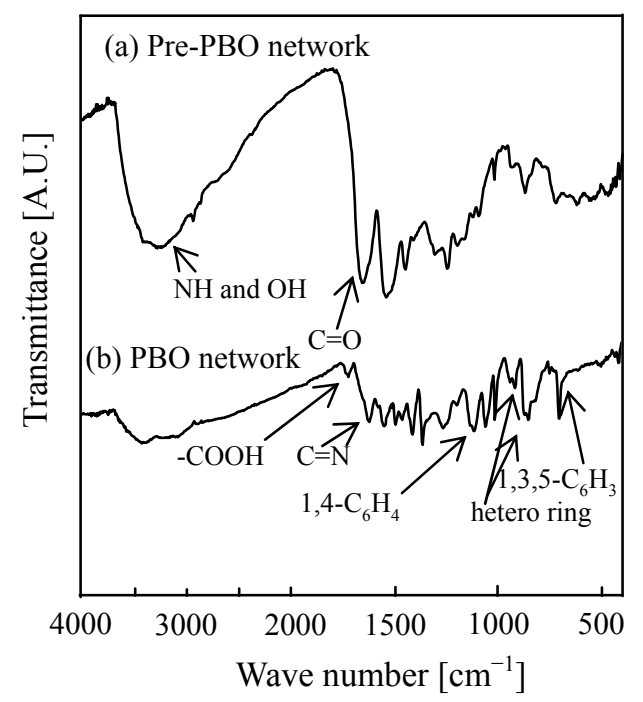

Fig. 5. IR spectra of(a) pre-PBO network, (b)PBOnetwork.
A water molecule was eliminated from the amide bond by heating the pre-PBO network and cyclization to an oxazole ring occurred. In this way, the PBO network was prepared. TGA was performed to estimate the temperature for the cyclization reaction. The results are shown in Fig. 6. Upon heating, a weight loss was observed at around $230^{\circ} \mathrm{C}$ due to the elimination of water associated with the cyclization reaction. No further weight loss was observed when the temperature was held at $350^{\circ} \mathrm{C}$, confirming completion of the reaction.

From the results, it was concluded that the temperature necessary for completion of the cyclization reaction was $350{ }^{\circ} \mathrm{C}$.

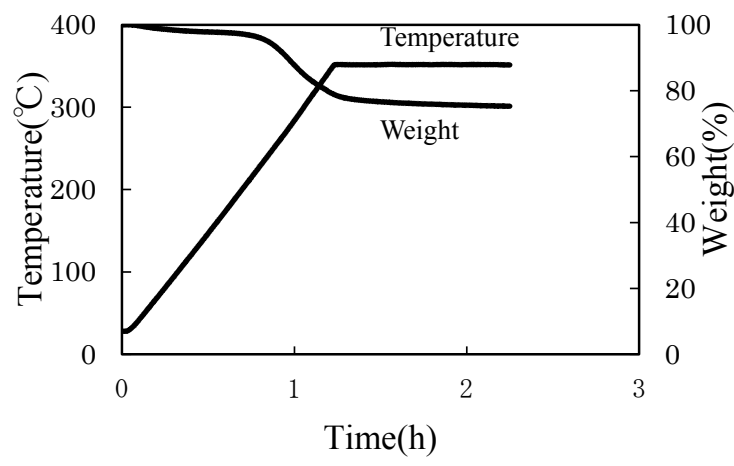

Fig. 6. Thermogravimetric analysis of pre-PBO network (TPC:TMC $=7: 3$ ) held at $350{ }^{\circ} \mathrm{C}$.

A PBO network was obtained by heat treatment of the pre-PBO network at $350{ }^{\circ} \mathrm{C}$, as shown in Fig. 7. In the IR spectrum of the PBO network after the cyclization reaction, the peak due to the amide bond characteristic of the pre-PBO network was no longer seen, and new absorption bands at $1620 \mathrm{~cm}^{-1}$ due to $\mathrm{C}=\mathrm{N}$ and at 920 and $860 \mathrm{~cm}^{-1}$ due to oxazole were observed. These results confirmed that a cyclization reaction occurred upon heat treatment of the pre-PBO network to form the desired oxazole-containing $\mathrm{PBO}$ network.

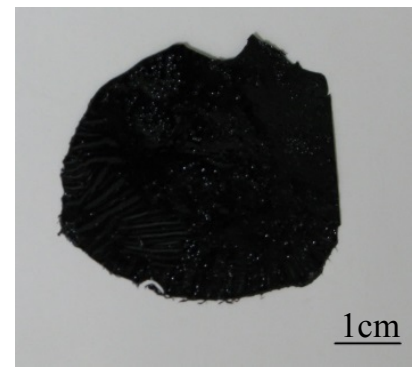

Fig. 7. PBO network. 
3.2. Structure and physical properties of the PBO network

The apparent density of the obtained PBO network was $0.82 \mathrm{~g} / \mathrm{cm}^{3}$. Its porosity was $46 \%$, calculated on the basis of the density of PBO fiber of $1.54 \mathrm{~g} / \mathrm{cm}^{3}$ [5].

The surface and fracture surface of the PBO network were observed by scanning electron microscopy (SEM), as shown in Fig. 8 No micrometer or submicrometer order voids were observed. Conversely, the capacity of micropores of diameter less than $1 \mathrm{~nm}$ was $0.059 \mathrm{~mL} / \mathrm{g}$, as determined by carbon dioxide adsorption measurement. This implied that a porous film containing many nanometer scale voids had been obtained.

TGA measurement at a heating rate of $5{ }^{\circ} \mathrm{C} / \mathrm{min}$ was performed to study the heat resistance of the obtained PBO network under nitrogen atmosphere, as shown in Fig. 9. The 10\% weight loss temperature was $575^{\circ} \mathrm{C}$, demonstrating that the PBO network showed very high heat resistance.

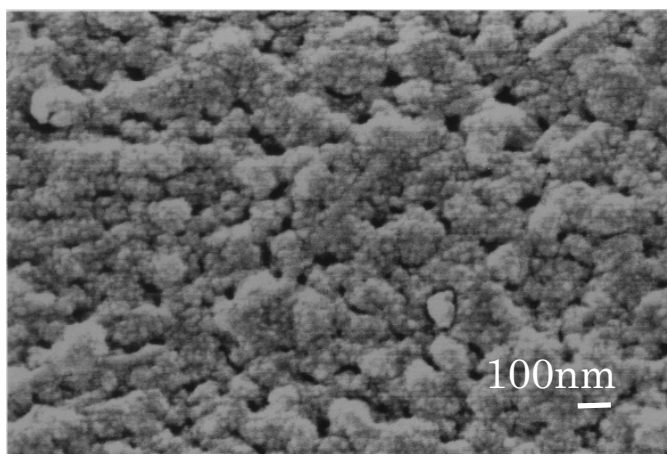

Fig. 8. SEM image of PBO network film

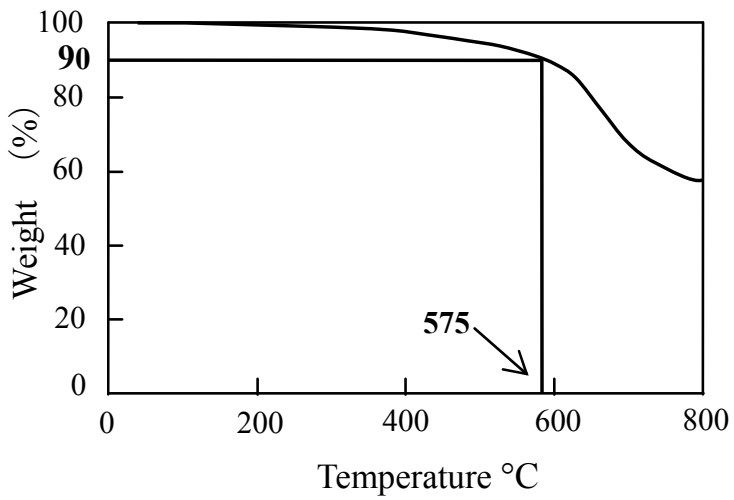

Fig. 9. TG of PBO network film.

Bending tests were performed to evaluate the mechanical properties of the obtained PBO network. The results of the bending test are shown in Table 1. The flexural modulus was 980 MPa.

Table 1. Bending test results for the PBO network.

\begin{tabular}{|c|c|c|c|}
\hline $\begin{array}{c}\text { Sample } \\
\text { thickness } \\
{[\mathrm{mm}]}\end{array}$ & $\begin{array}{c}\text { Modulus } \\
{[\mathrm{MPa}]}\end{array}$ & $\begin{array}{c}\text { Breaking } \\
\text { strength } \\
{[\mathrm{MPa}]}\end{array}$ & $\begin{array}{c}\text { Breaking } \\
\text { elongation } \\
{[\%]}\end{array}$ \\
\hline 0.61 & 980 & 7.8 & 6.3 \\
\hline
\end{tabular}

In addition, dynamic viscoelasticity measurements were performed to confirm the thermal stability of the mechanical properties. The results are shown in Fig. 10. It can be seen that the modulus of the PBO network hardly decreased up to at least $400{ }^{\circ} \mathrm{C}$.

The thermal coefficient of linear expansion of the PBO network film was measured as $(2.18 \pm$ $1.55) \times 10^{-5} /{ }^{\circ} \mathrm{C}$, which showed that the film had low thermal expansivity.

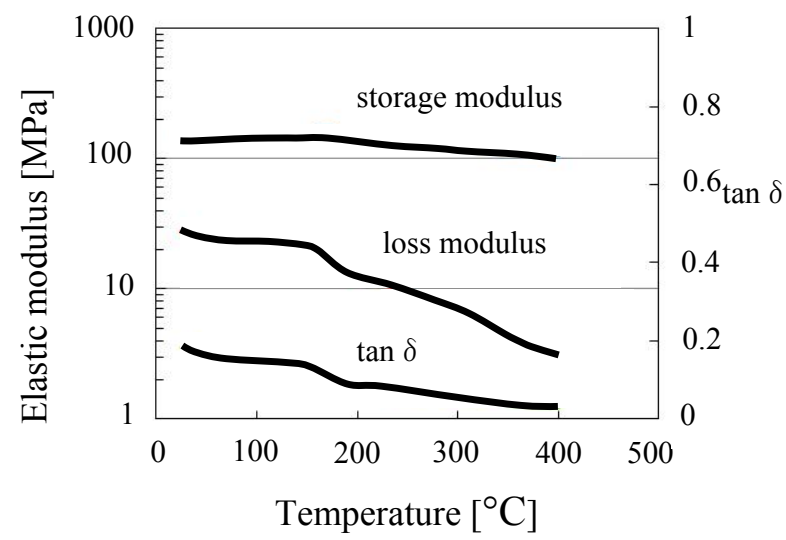

Fig.10. Viscoelasticity of PBO network film

Thermal diffusivity measurements of the obtained PBO network were performed in both the in-plane and thickness directions. The results obtained are shown in Table 2 . The value in the in-plane direction was $7.4 \times 10^{-7} \mathrm{~m}^{2} / \mathrm{s}$, somewhat larger than that in the thickness direction, $3.4 \times$ $10^{-7} \mathrm{~m}^{2} / \mathrm{s}$. We surmise that the molecular chains are oriented in the in-plane direction during molding. The results also suggest that the PBO network film may be applied as a thermal insulation material, because the thermal diffusivity is almost the same as that of polystyrene form, which is well known as a typical thermal insulation material. 
Table 2. Thermal diffusivities of the PBO network in the in-plane and thickness directions.

\begin{tabular}{|c|c|}
\hline & $\begin{array}{c}\text { Thermal diffusivity } \\
\left(\mathrm{m}^{2} / \mathrm{s}\right)\end{array}$ \\
\hline In-plane direction & $7.4 \times 10^{-7}$ \\
\hline Thickness direction & $3.4 \times 10^{-7}$ \\
\hline
\end{tabular}

3.3. Preparation of a highly heat-resistant PBO network film

Close inspection of the thermogravimetric trace in Fig. 9 revealed that the weight loss occurred in two steps. When the change in the PBO network structure associated with the weight loss was studied by IR spectroscopy, it was seen that the peak due to the unreacted carboxyl group disappeared at about $400{ }^{\circ} \mathrm{C}$. Therefore, the PBO network was heat-treated at $450{ }^{\circ} \mathrm{C}$ and the IR spectrum of the product was recorded, as shown in Fig. 11. The resulting spectrum confirmed disappearance of the peak due to the unreacted carboxyl group in the untreated PBO network.

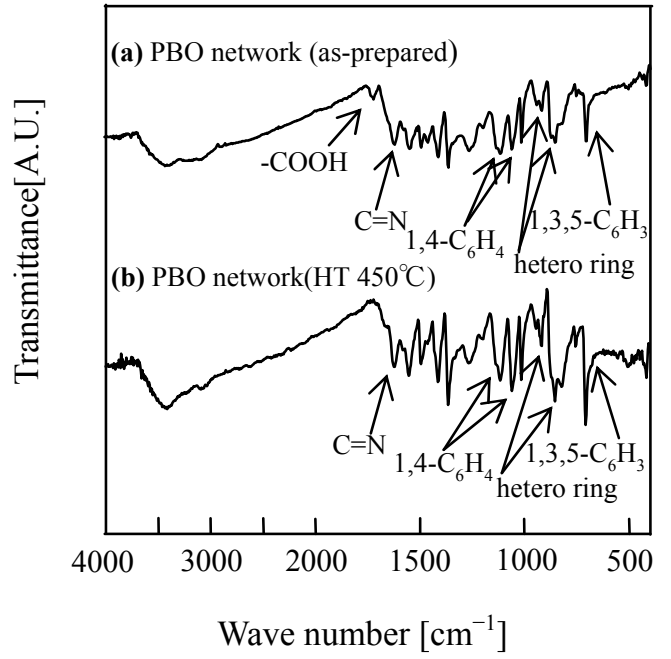

Fig. 11. IR spectra of the as-prepared PBO network and heat-treated PBO network film $\left(450{ }^{\circ} \mathrm{C}, 8 \mathrm{~h}\right)$.

The spectrum also indicated that only decarboxylation had occurred, because no changes in the other peaks due to the main chain of the PBO network were observed. TGA results for the PBO network after the heat treatment, as shown in Fig. 12, revealed that the $10 \%$ weight loss temperature had been raised to $623{ }^{\circ} \mathrm{C}$, indicating higher heat resistance than that of the untreated PBO network.

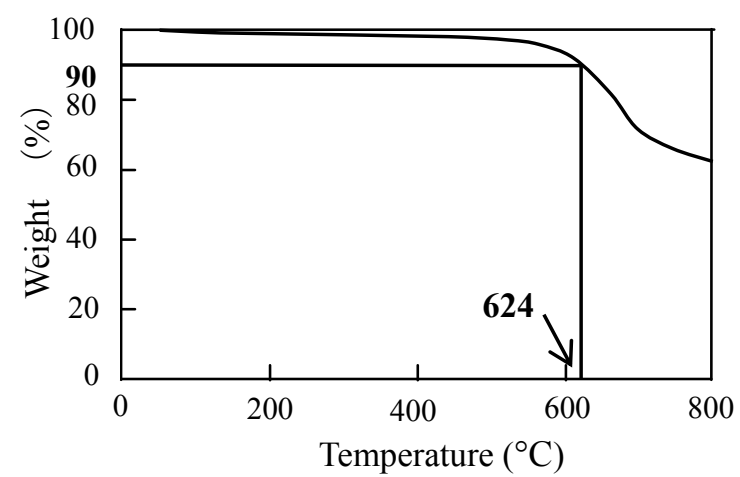

Fig. 12. TGA of heat-treated PBO network film $\left(450^{\circ} \mathrm{C}, 8 \mathrm{~h}\right)$.

\section{Conclusions}

-A novel method for synthesizing a PBO network through a pre-PBO network has been established.

- Many nanometer scale voids are present in the porous PBO network.

- The $10 \%$ weight loss temperature of the PBO network after heat treatment was $623{ }^{\circ} \mathrm{C}$, indicating high heat resistance.

- Dynamic viscoelasticity measurements revealed that the modulus of the PBO network was hardly changed, even on heating to $400{ }^{\circ} \mathrm{C}$.

- The PBO network showed low thermal diffusivity.

\section{References}

1. J.F.Wolfe, et al., Macromolecules, 14 (1981) 909.

2. T.Uchida, et al., Sen-I Gakkaishi, 59 (6) (2003) 207.

3. H.Nishino, Patent Application Publication No. $\mathrm{H} 2-247225$.

4. M.Hasegawa, et al., J. Photopolym. Sci. Technol., 17 (2) (2004) 253.

5. D.C.Martin, E.L.Thomas, Macromolecules, 24 (1991) 2450. 